\title{
INVESTIGACIONES
}

\section{Ajuste formación-empleo: el alumnado propone}

\author{
Training-employment match: students propose
}

\author{
Pilar Martínez Clares $^{a}$, Natalia González Morga ${ }^{a}$ \\ ${ }^{a}$ Departamento de Métodos de Investigación y Diagnóstico en Educación, Universidad de Murcia. \\ pmclares@um.es, natalia.gonzalez@um.es
}

\section{RESUMEN}

El debate sobre el binomio formación-empleo se intensifica en las actuales políticas educativas y económicas de la Unión Europea. Por ello, el presente trabajo valora la adecuación de la formación universitaria a los requerimientos laborales y su aplicación en las prácticas curriculares. Participan en la investigación 1137 estudiantes a través de un cuestionario diseñado ad hoc con una óptima fiabilidad y validez. Los resultados señalan un $28 \%$ de alumnado que percibe la formación bastante o muy adecuada con diferencias significativas entre ramas de conocimiento, mientras que el $42.2 \%$ percibe muy elevada la aplicación de dicha formación en las prácticas curriculares. Estos resultados concuerdan con otros estudios de egresados españoles como el de Michavila et al. (2016). Los participantes plantean una amplia variedad de propuestas dirigidas a la mejora de estos resultados y marcan líneas de cambio en la formación universitaria hacia su ajuste con los requerimientos profesionales.

Palabras clave: formación universitaria, mercado laboral, prácticas curriculares, propuestas de mejora.

\begin{abstract}
The debate on the training-employment pairing intensifies in the current educational and economic policies of the European Union. For this reason, this work assesses the adequacy of university training to work requirements, as well as its application in curricular practices. 1137 students participate in the research through an ad hoc designed questionnaire with optimum reliability and validity. The results indicate $28 \%$ of students who perceive the training to be fairly or very adequate with significant differences between areas of knowledge, while $42.2 \%$ perceive the application of such training in curricular practices to be very high. These results are in agreement with other studies of Spanish graduates such as the one by Michavila et al. (2016). Participants propose a wide variety of proposals aimed at improving these results and mark lines of change in university education towards their adjustment with professional requirements.
\end{abstract}

Key words: university training, labor market, curricular practices, proposals for improvement. 


\section{INTRODUCCIÓN}

Las demandas de adaptación del sistema educativo a las necesidades sociales y económicas de un país constituyen hoy el reto fundamental de las políticas educativas de la Unión Europea. Las declaraciones gubernamentales para la implantación del Plan Bolonia en las Universidades, el Marco de Cooperación Europea en el ámbito juvenil 2010-2018, la Estrategia Europa 2020 o el informe del Consejo Europeo del Grupo de Reflexión sobre el futuro de la UE en 2030, proponen capacitar a todas las personas para las transiciones laborales, en especial la transición a la vida activa, para aminorar los desequilibrios entre la oferta y la demanda de conocimientos. Para ello, se apuesta por el aprovechamiento del talento humano y la excelencia en todas las etapas educativas.

Organismos como la Organización Internacional del Trabajo (OIT, 2014), el Centro Europeo para el Desarrollo de la Formación Profesional (CEDEFOP, 2015, 2018), o la Organización para la Cooperación y el Desarrollo en Educación (OCDE, 2012, 2015) dedican gran parte de sus informes a esclarecer y profundizar en la relación de la Educación Superior y el mercado de trabajo y coinciden en señalar entre sus objetivos prioritarios, la adecuación de la formación a las necesidades del mercado productivo para potenciar el desarrollo económico y social.

Nace el concepto de desajuste competencial o skill mismach para CEDEFOP (2014) referido a la situación de desequilibrio en las competencias disponibles y las necesidades del mercado laboral. Este organismo, alude a este desajuste competencial cuando el entorno sociolaboral tiene dificultades para cubrir las vacantes con profesionales que tengan el perfil formativo adecuado; pero también, cuando los trabajadores aceptan puestos en los que no se aprovechan adecuadamente sus competencias y cualificaciones dando lugar al fenómeno de la sobrecualificación o la infracualificación.

$\mathrm{Si}$ a menudo este término es utilizado para describir dos formas de inestabilidad entre la oferta formativa (perfil formativo) y la demanda del mercado laboral (perfil profesional), en otras ocasiones se diferencia entre desajuste vertical, asociado a las distancias encontradas entre el nivel de estudios del graduado y el requerido en su puesto de trabajo y desajuste horizontal, donde el trabajador se adapta al nivel de estudios, pero las competencias adquiridas o área de conocimiento no responden al campo de trabajo o profesión desempeñado (Michavila, Martínez, Martín-González, García-Peñalvo y Cruz-Benito, 2016; Rodríguez Esteban, Vidal y Vieira, 2019). La economía del conocimiento fruto de la llegada de la digitalización en todos los ámbitos de nuestra vida es la impulsora de nuevas y diferentes formas en el mundo laboral, que a la vez crea y destruye empleos y modifican los perfiles de muchas ocupaciones. A este proceso de reconversión laboral, Schwab (2016) lo denomina la $4^{\circ}$ Revolución Industrial (4RI) e inspira el surgimiento del nuevo orden empresarial o era del empleo 4.0.

El contexto socioeconómico en permanente transformación requiere en los graduados una formación basada en competencias que desarrolle su capacidad de adaptación y aprendizaje continuo, pero también de profesores comprometidos con un nuevo enfoque metodológico y calidad del sistema (Hargreaves y Fulan, 2014; Ursin, 2017). El modelo educativo basado en la economía del conocimiento necesita modificar en profundidad cómo se enseña, y sobre todo, cómo y qué se aprende en las aulas universitarias, tarea compleja que afecta tanto a la cultura universitaria como al modo organizativo y pedagógico.

Los informes realizados hasta la fecha arrojan una visión desoladora sobre el rendimiento de las universidades para generar profesionales con una formación adecuada 
al crecimiento y evolución de la economía 4.0 en nuestro contexto. El Índice Europeo de Competencias (ESI, European Skills Index) elaborado por CEDEFOP (2018), valora la conexión de la cualificación de la fuerza de trabajo con las demandas laborales y entre los indicadores utilizados se encuentra el ajuste formativo al puesto de trabajo. Este estudio sitúa a España en penúltima posición entre los países de la UE, con una puntuación de 11 puntos sobre 100 en cuanto adecuación de las competencias de sus profesionales. La actualización de este informe (CEDEFOP, 2020) sigue mostrando la tendencia hacia el desajuste de competencias, aunque se especifica su especial escasez en las cualificaciones de ciencias y área de la salud. Estos datos alertan una situación compleja en el sistema universitario en pro de atender el dinamismo laboral, quizá debido a la dificultad que supone seguir el ritmo de los sistemas productivos cuando cambian a un ritmo casi vertiginoso (Teichler, 2015).

Los jóvenes españoles posen el nivel de estudios más elevado de la historia de España (más del $42 \%$ de entre 25 a 29 años han completado estudios superiores, un porcentaje superior a la media de la Unión Europea (UE), y casi el 30\% estudios universitarios) y sin embargo, se aprecian problemas para explotar la inversión en formación. Muchos universitarios no encuentran empleo y buena parte de los que consiguen ocupan trabajos que no se corresponden con su formación (Pérez García, 2018), tan solo 13 de cada 100 graduados consigue un empleo adecuado a su formación (Bartlett y Uvalic, 2019).

El ajuste de la educación universitaria es un indicador de rendimiento formativo y a la vez favorece la empleabilidad de los graduados, está condicionado en muchos casos, no solo por diferentes factores que quedan al margen del control de las universidades, sino también, por el propio funcionamiento del sistema formativo en su conjunto.

Desde la visión de los empleadores, los sistemas de formación europeos siguen mostrando deficiencias a la hora de proporcionar las competencias adecuadas para la inserción profesional, declarando que la experiencia educativa se aleja de la realidad del entorno laboral (Franke, 2016). Además, reiteran la dificultad para cubrir los puestos de trabajo con candidatos que tengan las competencias adecuadas, aludiendo a la excesiva carga de contendido teórico que proporcionan las instituciones educativas, con escasa aplicación real en la práctica (García-Aracil y Van der Velden, 2008; Palmer, Montaño y Palou, 2009; Humberg, Van der Valden, y Verhagen, 2013; CEDEFOP, 2014).

De igual forma, los estudiantes también comparten esta visión, percibiendo una excesiva burocracia y programas académicos obsoletos y distanciados del desempeño del trabajo real (Alonso, Fernández y Nyssen, 2009).

Ante la distancia entre la formación que reciben los graduados en las aulas y los requerimientos laborales, las universidades han de reaccionar y reorientarse hacia el tejido productivo bajo un modelo formativo basado en competencias, entendiendo como un factor de la calidad, el equilibrio entre este binomio indisociable (formación y empleo). Desde esta premisa, además de existir argumentos suficientes que avalan la necesidad de proseguir la investigación sobre el tema, el escenario universitario es propicio para repensar sobre sus resultados y así poder atender y plantear nuevas propuestas que optimicen dicha situación.

$\mathrm{Si}$ es frecuente encontrar estudios que valoran este desajuste desde la visión de los empleadores o una vez que los graduados ya están insertados en el mercado de trabajo (Michavila et al., 2016; Bartlett y Uvalić, 2019), es menos habitual que estos se ubiquen en las propias aulas universitarias, escuchando la voz de sus protagonistas, el alumnado. Concretamente el objetivo de este trabajo es triple: 
- Conocer y analizar la adecuación de la formación universitaria a los requerimientos laborales de los graduados de las distintas ramas de conocimiento.

- Conocer y contrastar la aplicación de la formación recibida en las prácticas curriculares de los graduados en relación de la rama de conocimiento y el ámbito profesional elegido.

- Identificar y analizar las propuestas de mejora para la adecuación de la formación a los requerimientos laborales.

\section{METODOLOGÍA}

\subsection{DISEÑO Y PARTICIPANTES}

La investigación se ha llevado a cabo desde un diseño no experimental, descriptivo y transversal en 19 títulos de Grado de la Universidad de Murcia. La población objeto de estudio corresponde al alumnado de $4^{\circ}$ curso de las cinco ramas de conocimiento.

Tras realizar un muestreo probabilístico estratificado se obtiene una muestra de 1137 estudiantes con un nivel de confianza de $99 \%$ y un error muestral de $3.44 \%$, respetando la proporcionalidad del alumnado en cada uno de los estratos o áreas de conocimiento (tabla 1).

Tabla 1. Distribución de los participantes por títulos y ramas de conocimiento

\begin{tabular}{|c|c|c|c|c|c|}
\hline $\begin{array}{c}\text { Rama de } \\
\text { conocimiento }\end{array}$ & $\begin{array}{l}\text { Títulos de Grado } \\
\text { participantes }\end{array}$ & $\begin{array}{c}\mathbf{N}^{\circ} \text { de } \\
\text { participantes }\end{array}$ & $\begin{array}{c}\text { Total de } \\
\text { participantes } \\
\text { por rama }\end{array}$ & $\begin{array}{c}\% \text { de muestra } \\
\text { estratificada } \\
\text { recomendada } \\
n=1137\end{array}$ & $\begin{array}{c}\text { \% de muestra } \\
\text { estratificada } \\
\text { alcanzada }\end{array}$ \\
\hline \multirow{4}{*}{$\begin{array}{c}\text { Ciencias } \\
\text { Sociales (CSS) }\end{array}$} & Pedagogía & 78 & \multirow{4}{*}{565} & \multirow{4}{*}{$54 \%$} & \multirow{4}{*}{$50 \%$} \\
\hline & Educación Social & 62 & & & \\
\hline & Educación Primaria & 313 & & & \\
\hline & Educación Infantil & 112 & & & \\
\hline \multirow{3}{*}{$\begin{array}{l}\text { Ciencias de la } \\
\text { Salud (CS) }\end{array}$} & Logopedia & 28 & \multirow{3}{*}{181} & \multirow{3}{*}{$18 \%$} & \multirow{3}{*}{$16 \%$} \\
\hline & Enfermería & 98 & & & \\
\hline & Psicología & 55 & & & \\
\hline \multirow{5}{*}{$\begin{array}{c}\text { Ciencias } \\
\text { Experimentales } \\
(\mathrm{CE})\end{array}$} & $\begin{array}{l}\text { Ciencias } \\
\text { Ambientales }\end{array}$ & 43 & \multirow{5}{*}{159} & \multirow{5}{*}{$9 \%$} & \multirow{5}{*}{$14 \%$} \\
\hline & Biología & 40 & & & \\
\hline & Física & 16 & & & \\
\hline & Bioquímica & 52 & & & \\
\hline & Matemáticas & 8 & & & \\
\hline
\end{tabular}




\begin{tabular}{|c|c|c|c|c|c|}
\hline \multirow{6}{*}{$\begin{array}{c}\text { Artes y } \\
\text { humanidades } \\
(\mathrm{AH})\end{array}$} & Historia del Arte & 26 & \multirow{6}{*}{181} & \multirow{6}{*}{$17 \%$} & \multirow{6}{*}{$16 \%$} \\
\hline & Geografía & 28 & & & \\
\hline & Traducción Francés & 10 & & & \\
\hline & Traducción Inglés & 26 & & & \\
\hline & Estudios Ingleses & 38 & & & \\
\hline & Historia & 53 & & & \\
\hline $\begin{array}{l}\text { Ingenierías } \\
\text { (ING) }\end{array}$ & Informática & 51 & 51 & $2 \%$ & $4 \%$ \\
\hline
\end{tabular}

\subsection{INSTRUMENTO Y VARIABLES DE ESTUDIO}

La recogida de datos se realiza a través de un cuestionario diseñado ad hoc que valora el ajuste formativo con las demandas del mercado laboral desde distintas variables. Para los propósitos de este trabajo se analizan las siguientes cuestiones:

- Variables cuantitativas: grado de adecuación de la formación recibida a lo largo de la carrera a los requerimientos del mercado laboral; la aplicación de las competencias desarrolladas en su experiencia profesional relativa al periodo de prácticas curriculares externas; y el ámbito laboral elegido para desarrollarse profesionalmente. La valoración de los estudiantes se realiza en una escala de valoración ordinal con cinco opciones de respuesta $(1=$ nada, $2=$ poco, $3=$ regular, $4=$ bastante y $5=$ mucho) y la variable ámbito profesional en una escala nominal cuyas categorías de respuesta son: educativo, comunitario, empresarial, otros.

- Variables cualitativas: propuestas de mejorar hacia el ajuste de la formación universitaria a las demandas laborales actuales.

La fiabilidad del cuestionario global del que forma parte estas variables denominado CECTGRA, ha sido analizada con Alfa de Cronbach, obteniendo un coeficiente de alfa $=.927$. Su estructura interna fue validada a través de procedimiento de juicio de expertos y métodos de agregados individuales, y además cada una de las escalas de valoración de competencias que integra a través de Análisis Factorial Confirmatorio, obteniendo unos indicadores de bondad de ajuste satisfactorios con valores que oscilan entre NFI=.903.951; CFI=.916-.962; RMSEA=.074-.057) (Martínez Clares y González Morga, 2018b).

\subsection{ANÁLISIS DE LOS DATOS}

Una vez recogida la información, el análisis de los datos cuantitativo se realiza con la estadística descriptiva e inferencial. Se recurre a la prueba paramétricas ANOVA de un factor dado el cumplimiento del principio de normalidad de los datos en las preguntas objeto de estudio y el de homocedasticidad, ejecutadas con el programa SPSS v23.

Para contrastar la percepción sobre la adecuación y aplicabilidad de la formación en las distintas ramas de conocimiento se ha aplicado el Análisis de Varianza (ANOVA), 
asumiendo un nivel de significación del 5\% (p<.05). Para profundizar en las diferencias detectadas dentro del intervalo de confianza ( $\mathrm{IC}=95 \%$ ), se ha efectuado el cálculo complementario post hoc, recurriendo al estadístico Gabriel que controla el error de nivel I y además, el más apropiado para contrastar grupos de tamaño muestral diferente, como es el caso de este estudio.

El análisis de datos cualitativo se realiza con el programa Atlas ti 7 y través de un proceso que pasa por diferentes momentos: reducción de datos, disposición y transformación de los datos, y obtención de resultados y verificación de conclusiones (Rodríguez, Lorenzo y Herrera, 2005). La figura 1 representa de forma gráfica este proceso.

Figura 1. Proceso de análisis de datos cualitativos. Adaptado de Rodríguez et al. (2005)

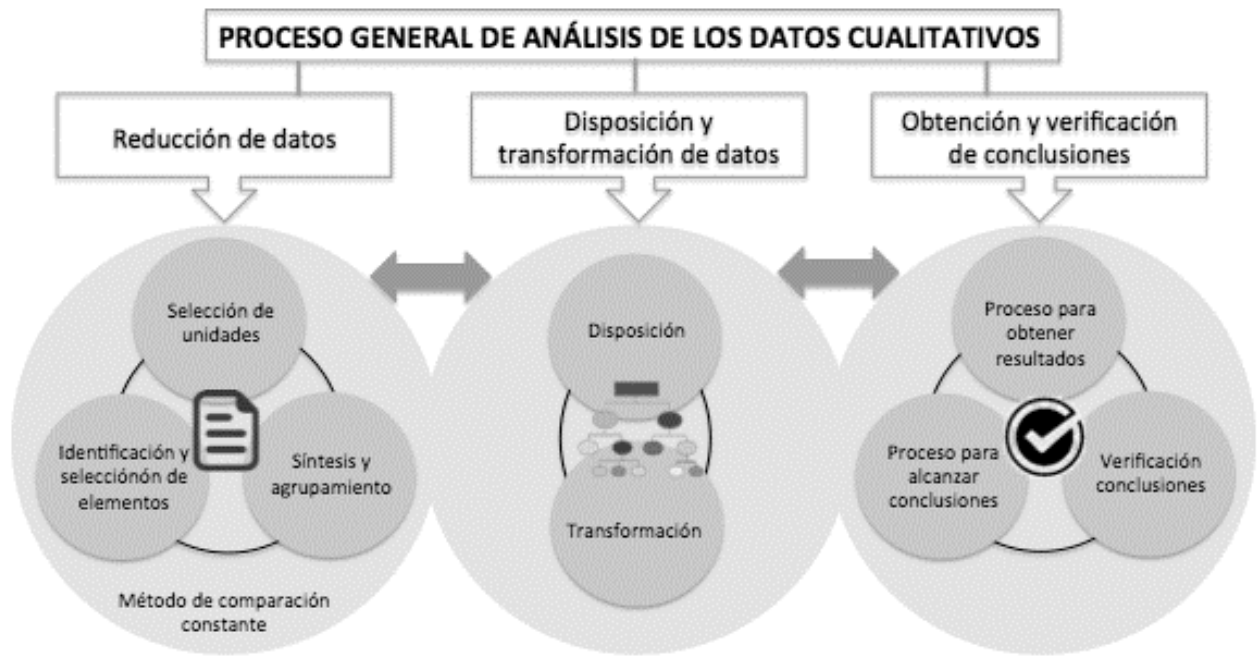

- Reducción de los datos: Esta fase consiste en la división de la información en unidades de significado en base en base a un criterio temático (el texto queda divido en función de los diferentes temas tratados). Una vez clasificadas las unidades de análisis, se pasa a identificarlas y etiquetarlas; establecer categorías y códigos de una forma inductiva y a través del método de comparación constante entre unidades de significado (Osses, Sánchez e Ibáñez, 2006) en busca de similitudes y diferencias entre ellas. La lista final de códigos, subcategorías y categorías se cierra cuando han sido teóricamente saturadas, es decir, de la información no emerge ningún dato nuevo.

- Disposición y tratamiento de datos: Establecidos los códigos el siguiente paso es elegir un procedimiento de disposición y transformación de dichos datos. En este trabajo se opta por los diagramas o redes semánticas para permitir visualizar las relaciones entre conceptos (Strauss y Corbin, 2002). En ellas, se representan los aspectos básicos y relevantes interrelacionados entre sí, de cuya interpretación se 
obtiene los resultados de la investigación (describen y explican el problema investigado).

- Obtención de resultados y verificación de conclusiones: Esta última fase hace referencia a la interpretación de la información apoyada en fragmentos narrativos. La verificación de los resultados se realizar con el intercambio o comparación de los resultados con otros estudios.

\section{RESULTADOS}

\subsection{ADECUACIÓN DE LA FORMACIÓN A LAS DEMANDAS LABORALES}

El 28\% de los estudiantes consideran adecuada su formación universitaria a las demandas de su perfil profesional, mientras el 50\% reconoce un ajuste regular o suficiente, y el $22 \%$ restante muestra su escasez e incluso inexistencia (figura 2). De forma general, la puntuación media de todo el alumnado es de 3.04 (DT= 0.879), lo que manifiesta una adecuación media o regular.

Figura 2. ¿Crees que la formación que has recibido se ajusta al requerimiento del mercado laboral? Porcentaje global.

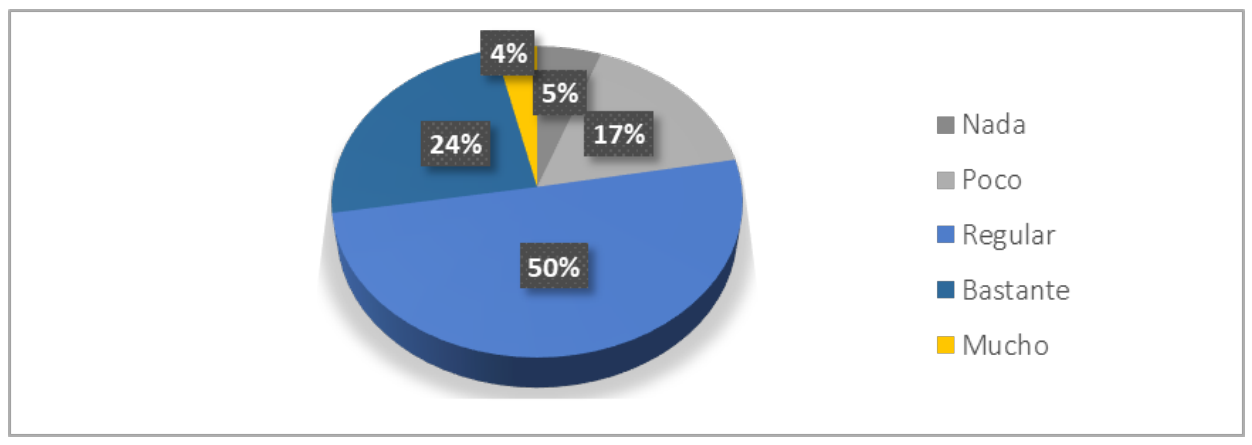

Estas respuestas varían en función de la rama de conocimiento. Mientras los estudiantes de Ciencias de la Salud e Ingeniería son los que perciben una mayor adecuación entre el perfil formativo y profesional donde más del $50 \%$ de los estudiantes considera que existe bastante o mucha, el alumnado de Artes y Humanidades obtienen la puntuación más baja, el $35.7 \%$ reconoce un escaso o nulo ajuste entre su formación universitaria y los requerimientos del mercado de trabajo (figura 3). En relación al alumnado de Ciencias Sociales y Ciencias Experimentales, solo un $19.7 \%$ y $18.2 \%$ respectivamente, manifiestan un ajuste adecuado. 
Figura 3. ¿Crees que la formación que has recibido se ajusta a los requerimientos del mercado laboral? Porcentajes por rama de conocimiento.

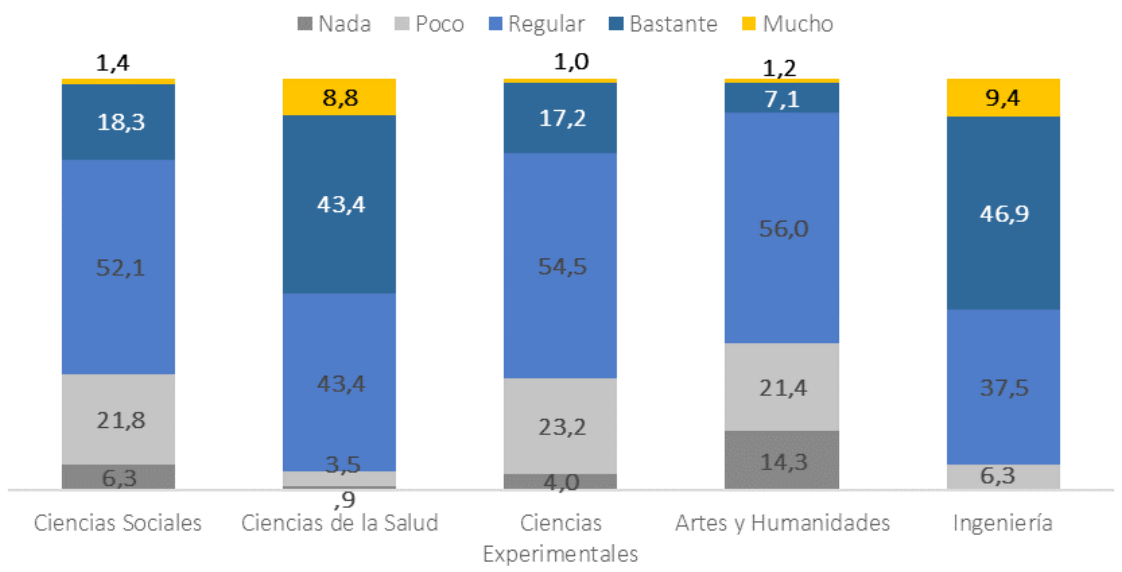

Para confirmar la existencia de diferencias significativas en la adecuación de la formación a los requerimientos profesionales en función de la rama de conocimiento se recurre al Análisis de la Varianza, previo análisis del principio de homocedasticidad de los datos mediante la prueba de Levene. Esta prueba estadística informa de la existencia de una varianza similar entre los datos de los diferentes grupos $(p=.347)$ y por tanto adecuada para recurrir el estadístico ANOVA. Los resultados muestran diferencias en la percepción de adecuación de la formación en función de la rama de conocimiento a la que pertenece el alumnado $[\mathrm{F}(4)=6.378, \mathrm{p}=.000]$. Se recurre a las pruebas Post hoc para conocer entre qué ramas son significativas y se observa como la significatividad de las diferencias son generalizables entre todas las ramas de conocimiento, excepto entre Ciencias Sociales, Ciencias Experimentales y Artes y Humanidades, donde los estadísticos muestran una percepción de adecuación formativa similar entre áreas de conocimiento, tal y como muestra la tabla 2.

Tabla 2. Diferencias significativas entre ramas de conocimiento ¿Crees que la formación que has recibido se adecúa a los requerimientos del mercado laboral? ANOVA. Variable dependiente

\begin{tabular}{|c|c|c|c|c|c|c|}
\hline $\begin{array}{l}\text { (I) Área de } \\
\text { conocimiento }\end{array}$ & Media & DT & (J) Área de conocimiento & $\begin{array}{l}\text { Diferencia de } \\
\text { medias (I-J) }\end{array}$ & $\begin{array}{c}\text { Error } \\
\text { estándar }\end{array}$ & Sig. \\
\hline \multirow{4}{*}{$\begin{array}{l}\text { Ciencias } \\
\text { Sociales }\end{array}$} & \multirow{4}{*}{2,87} & \multirow{4}{*}{, 836} & Ciencias de la Salud &,$- 691^{*}$ & ,101 & 000 \\
\hline & & & Ciencias Experimentales &,- 013 & ,105 & 1,000 \\
\hline & & & Artes y Humanidades & 271 &, 110 & 144 \\
\hline & & & Ingeniería &,$- 728^{*}$ & 157 & 000 \\
\hline \multirow{4}{*}{$\begin{array}{l}\text { Ciencias de la } \\
\quad \text { Salud }\end{array}$} & \multirow{4}{*}{3,56} & \multirow{4}{*}{, 743} & Ciencias Sociales & ,691* & ,101, & 000, \\
\hline & & & Ciencias Experimentales & ,679* &, 110 & 000 \\
\hline & & & Artes y Humanidades & ,962* &, 115 & ,000 \\
\hline & & & Ingeniería &,- 036 & ,161 & 1,000 \\
\hline
\end{tabular}




\begin{tabular}{|c|c|c|c|c|c|c|}
\hline \multirow{4}{*}{$\begin{array}{c}\text { Ciencias } \\
\text { Experimentales }\end{array}$} & \multirow{4}{*}{2,88} & \multirow{4}{*}{,773 } & Ciencias Sociales &, 013 &, 105 & 1,000 \\
\hline & & & Ciencias de la Salud &,$- 679^{*}$ &, 110 & 000 \\
\hline & & & Artes y Humanidades &, 284 &, 119 &, 175 \\
\hline & & & Ingeniería &,$- 715^{*}$ &, 163 & 000 \\
\hline \multirow{4}{*}{$\begin{array}{c}\text { Artes y } \\
\text { Humanidades }\end{array}$} & \multirow{4}{*}{2,60} & \multirow{4}{*}{,866 } & Ciencias Sociales &,- 271 &, 110 & , 144 \\
\hline & & & Ciencias de la Salud &,$- 962^{*}$ &, 115 &, 000 \\
\hline & & & Ciencias Experimentales &,- 284 &, 119 &, 175 \\
\hline & & & Ingeniería &,$- 999^{*}$ &, 167 & 000 \\
\hline \multirow{4}{*}{ Ingeniería } & \multirow{4}{*}{3,59} & \multirow{4}{*}{,756 } & Ciencias Sociales &, $728^{*}$ &, 157 &, 000 \\
\hline & & & Ciencias de la Salud &, 036 &, 161 & 1,000 \\
\hline & & & Ciencias Experimentales &, $715^{*}$ &, 163 &, 000 \\
\hline & & & Artes y Humanidades & ,999* & ,167 & 000 \\
\hline
\end{tabular}

\subsection{APLICACIÓN DE LA FORMACIÓN EN LAS PRÁCTICAS CURRICULARES EXTERNAS}

Los alumnos de $4^{\circ}$ curso han tenido durante el grado contacto con su futuro profesional, y este indicador permite de nuevo, valorar el ajuste de la formación universitaria que reciben.

El $37 \%$ de los participantes considera que la aplicación de la formación recibida a la práctica curriculares es media (regular), mientras que el $20.6 \%$ revela que es poco o nada aplicable, sin embargo, el $42.2 \%$ restante la considera bastante o muy aplicable. De forma general, perciben una aplicación de sus competencias superior a la adecuación formativa, con un valor medio de 3.27 (DT=0.977).

Figura 3. Análisis descriptivo. Aplicabilidad de la formación universitaria en las prácticas curriculares-externas.

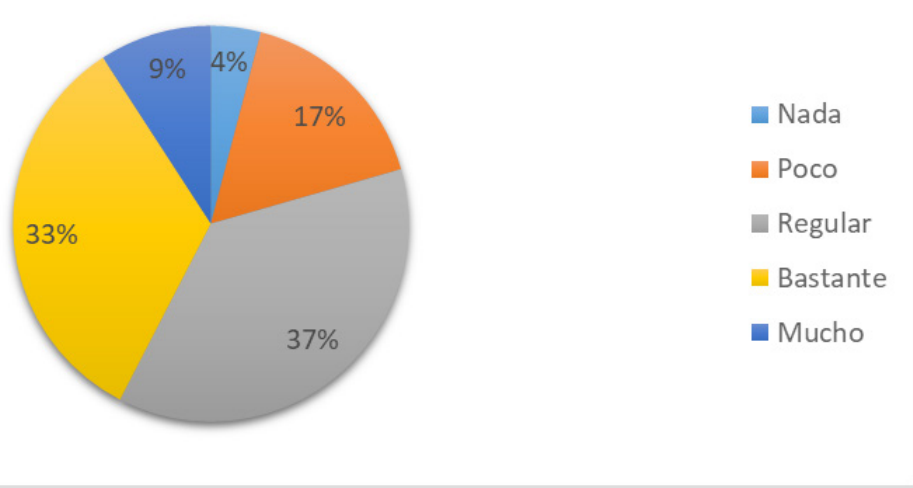


Si analizamos esta variable en función de la rama de conocimiento, se aprecian medias muy similares, siendo los estudiantes de Ingeniería los que obtienen la puntuación media más alta $(\bar{X}=3.57, \mathrm{DT}=1.005)$. Por el contrario, los estudiantes de Artes y Humanidades son lo que consideran su formación menos aplicable respecto al resto de ramas de conocimiento con una media de $\bar{X}=3.13$ (DT=1.049), aun así, ninguna rama de conocimiento baja de una $\bar{X}=$ de 3 (figura 3 ).

Estas diferencias entre áreas de conocimiento son significativas según la prueba paramétrica ANOVA de un factor, aplicada de nuevo una vez comprobado el cumplimiento del principio de homocedasticidad a través de la prueba de Levene (varianza similar entre los datos de los diferentes grupos, $p=.335$ ).

Los resultados muestran diferencias en la aplicabilidad de la formación en función de la rama de conocimiento a la que pertenece el alumnado $[\mathrm{F}(4)=4.629, p=.001]$. La prueba post hoc identifica estas diferencias estadísticamente significativas entre los estudiantes de Ingeniería y Artes y Humanidades, tal y como se aprecia en la tabla 3.

Tabla 3. Variable dependiente: ¿Cómo valoras la aplicabilidad de la formación universitaria en tu futuro desempeño profesional? ANOVA

\begin{tabular}{|c|c|c|c|c|c|c|}
\hline $\begin{array}{l}\text { (I) Área de } \\
\text { conocimiento }\end{array}$ & Media & DT & (J) Área de conocimiento & $\begin{array}{c}\text { Diferencia de } \\
\text { medias (I-J) }\end{array}$ & $\begin{array}{l}\text { Error } \\
\text { estándar }\end{array}$ & Sig. \\
\hline \multirow{4}{*}{$\begin{array}{l}\text { Ciencias } \\
\text { Sociales }\end{array}$} & \multirow{4}{*}{3,20} & \multirow{4}{*}{,948 } & Ciencias de la Salud &,- 219 & ,086 &, 115 \\
\hline & & & Ciencias Experimentales &,- 219 & 092 &, 171 \\
\hline & & & Artes y Humanidades & 074 & 085 & 1,000 \\
\hline & & & Ingeniería &,- 366 & 142 & 100 \\
\hline \multirow{4}{*}{$\begin{array}{l}\text { Ciencias de la } \\
\text { Salud }\end{array}$} & \multirow{4}{*}{3,42} & \multirow{4}{*}{,978 } & Ciencias Sociales & ,219 & 086 &, 115 \\
\hline & & & Ciencias Experimentales &,- 001 &, 112 & 1,000 \\
\hline & & & Artes y Humanidades & 292 & ,106 & ,060 \\
\hline & & & Ingeniería &,- 148 & , 156 & 1,000 \\
\hline \multirow{4}{*}{$\begin{array}{c}\text { Ciencias } \\
\text { Experimentales }\end{array}$} & \multirow{4}{*}{3,42} & \multirow{4}{*}{,937 } & Ciencias Sociales & ,219 & ,092 & , 171 \\
\hline & & & Ciencias de la Salud & ,001 &, 112 & 1,000 \\
\hline & & & Artes y Humanidades & ,293 &, 111 & 083 \\
\hline & & & Ingeniería &,- 147 & , 159 & 1,000 \\
\hline \multirow{4}{*}{$\begin{array}{c}\text { Artes y } \\
\text { Humanidades }\end{array}$} & \multirow{4}{*}{3,13} & \multirow{4}{*}{1,049} & Ciencias Sociales &,- 074 & 085 & 1,000 \\
\hline & & & Ciencias de la Salud &,- 292 & , 106 & 060 \\
\hline & & & Ciencias Experimentales &,- 293 &, 111 & 083 \\
\hline & & & Ingeniería &,$- 440^{*}$ &, 155 & ,046 \\
\hline \multirow{4}{*}{ Ingeniería } & \multirow{4}{*}{3,57} & \multirow{4}{*}{1,005} & Ciencias Sociales & ,366 & , 142 & 100 \\
\hline & & & Ciencias de la Salud & 148 & 156 & 1,000 \\
\hline & & & Ciencias Experimentales & , 147 &, 159 & 1,000 \\
\hline & & & Artes y Humanidades & , 440* & 155 & ,046 \\
\hline
\end{tabular}


La aplicación de la formación no solo varía en función de la rama de conocimiento, sino también en relación al ámbito laboral elegido para desarrollarse profesionalmente $(\mathrm{F}=2.062 ; \mathrm{gl}(4) ; \mathrm{p}=0.014)$. De forma general, los estudiantes perciben mayor aplicabilidad en el ámbito comunitario con un valor medio de $3.55(\mathrm{DT}=0.909)$ frente al ámbito educativo donde perciben un ajuste del perfil competencial más bajo $\left(\mathrm{X}^{-}=3.21, \mathrm{DT}=0.97\right)$. No obstante, hay que tener en cuenta que la elección del ámbito profesional difiere de una rama de conocimiento a otra, de ahí que en primer lugar se analicen las preferencias de los estudiantes para su desarrollo profesional en cada una de las opciones o categorías (figura 4).

Figura 4. Elección de ámbito profesional por rama de conocimiento. Porcentajes.

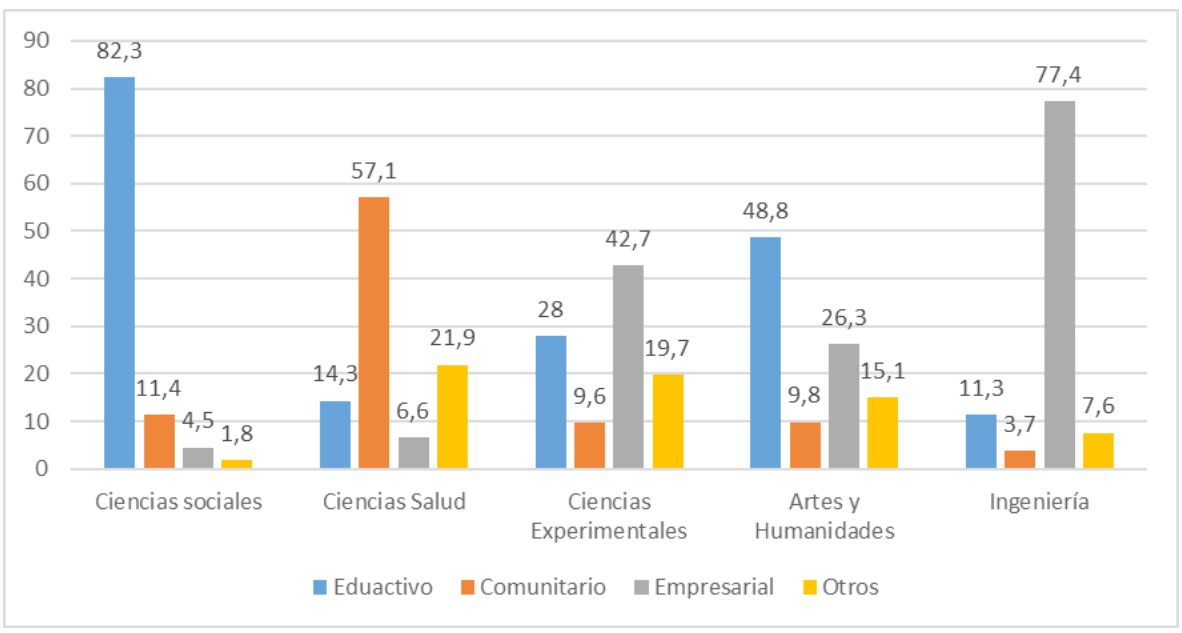

Mientras los estudiantes de Ciencias Sociales y Artes y Humanidades optan por el desarrollo de su carrera profesional en el ámbito educativo, los ingenieros y estudiantes de Ciencias Experimentales eligen el contexto organizativo (la empresa privada). Son los pertenecientes a las Ciencias de la Salud los que optan por el ámbito comunitario.

Ponderada la elección del ámbito profesional por parte del alumno de las distintas áreas de conocimiento, se relaciona esta percepción con la aplicabilidad de su formación para el desempeño de su carrera en cada uno de los ámbitos profesionales. 
Tabla 4. Datos estadísticos de la aplicación de la formación universitaria por ámbito profesional y en función de la rama de conocimiento. Kruskal Wallis

\begin{tabular}{|c|c|c|c|c|c|c|c|c|c|c|}
\hline \multirow[t]{2}{*}{$\begin{array}{c}\text { Ámbito } \\
\text { profesional }\end{array}$} & \multicolumn{2}{|c|}{$\begin{array}{c}\text { Ciencias } \\
\text { sociales }\end{array}$} & \multicolumn{2}{|c|}{$\begin{array}{l}\text { Ciencias } \\
\text { Salud }\end{array}$} & \multicolumn{2}{|c|}{$\begin{array}{c}\text { Ciencias } \\
\text { Experimentales }\end{array}$} & \multicolumn{2}{|c|}{$\begin{array}{c}\text { Artes y } \\
\text { Humanidades }\end{array}$} & \multicolumn{2}{|c|}{ Ingeniería } \\
\hline & $\bar{X}$ & DT & $\bar{X}$ & DT & $\bar{X}$ & DT & $\bar{X}$ & DT & $\bar{X}$ & DT \\
\hline Educativo & 3,19 & 0,942 & 3,28 & 0,958 & 3,48 & 1,061 & 3,16 & 1,108 & 3,50 & 1,000 \\
\hline Comunitario & 3,38 & 0,868 & 3,67 & 0,885 & 3,64 & 0,924 & 3,20 & 1,033 & 3,50 & 2,121 \\
\hline Empresarial & 3,33 & 1,500 & 3,43 & 0,976 & 3,22 & 0,815 & 3,31 & 1,151 & 3,50 & 1,007 \\
\hline Otros & 3,00 & 0,000 & 2,95 & 1,064 & 3,67 & 0,920 & 2,83 & 0,707 & 4,33 & 0,577 \\
\hline$F$ & \multicolumn{2}{|c|}{1.012} & \multicolumn{2}{|c|}{2.095} & \multicolumn{2}{|c|}{0.835} & \multicolumn{2}{|c|}{0.428} & \multicolumn{2}{|c|}{0.464} \\
\hline$g l$ & \multicolumn{2}{|c|}{3} & \multicolumn{2}{|c|}{3} & \multicolumn{2}{|c|}{3} & \multicolumn{2}{|c|}{3} & \multicolumn{2}{|c|}{3} \\
\hline Sig. $(p)$ & \multicolumn{2}{|c|}{.429} & \multicolumn{2}{|c|}{.033} & \multicolumn{2}{|c|}{.835} & \multicolumn{2}{|c|}{942} & \multicolumn{2}{|c|}{.801} \\
\hline Chi-cuadrado & \multicolumn{2}{|c|}{1,804} & \multicolumn{2}{|c|}{13,699} & \multicolumn{2}{|c|}{5,589} & \multicolumn{2}{|c|}{2,610} & \multicolumn{2}{|c|}{2,085} \\
\hline$g l$ & \multicolumn{2}{|c|}{3} & \multicolumn{2}{|c|}{3} & \multicolumn{2}{|c|}{3} & \multicolumn{2}{|c|}{3} & \multicolumn{2}{|c|}{3} \\
\hline Sig. $(p)$ & \multicolumn{2}{|c|}{0,614} & \multicolumn{2}{|c|}{0,003} & \multicolumn{2}{|c|}{0,133} & \multicolumn{2}{|c|}{0,456} & \multicolumn{2}{|c|}{0,555} \\
\hline
\end{tabular}

Los estudiantes de Ciencias de la Salud $(\bar{X}=3.67)$ junto con los estudiantes de Ciencias Sociales $(\bar{X}=3.38)$ muestran el grado más alto de aplicabilidad de su formación en el ámbito comunitario, mientras los estudiantes de Artes y Humanidades perciben esta aplicabilidad en el ámbito empresarial $(\bar{X}=3.31)$ y los alumnos de Ingeniería y Ciencias experimentales, en otros ámbitos profesionales sin especificar $(\bar{X}=4.33$ y $\bar{X}=3.67)$. Sin bien, los estadísticos reflejados en la tabla 4 , muestra tan solo diferencias significativas en Ciencias de la Salud $(\mathrm{p}=.003)$.

\subsection{PROPUESTAS DE MEJORA EN EL AJUSTE ENTRE FORMACIÓN-MERCADO LABORAL}

Una vez realizado el análisis cuantitativo del ajuste formativo, se analizan las respuestas cualitativas de los participantes sobre cómo mejorar la formación universitaria para ajustarla a las actuales demandas socioprofesionales. Las propuestas emitidas se clasifican en tres grandes categorías: La referida a la estructura del plan de estudios, una segunda relacionada con la planificación del proceso enseñanza/aprendizaje y la ultima la relativa a la orientación profesional a nivel institucional (tabla 5). 
Tabla 5. Categorías y códigos de las propuestas de mejora para ajustar la formación a las demandas profesionales

\begin{tabular}{|c|c|c|}
\hline \multicolumn{3}{|c|}{$\begin{array}{l}\text { PROPUESTAS DE MEJORA PARA AJUSTAR LA FORMACIÓN A LAS DEMANDAS } \\
\text { PROFESIONALES }\end{array}$} \\
\hline \multirow{10}{*}{$\begin{array}{l}\text { Categoría } 1.1 . \\
\text { Estructura del } \\
\text { plan de estudios }\end{array}$} & Código & Descripción \\
\hline & $\begin{array}{l}\text { Grupos más } \\
\text { reducidos }\end{array}$ & Menor número de alumnos por aula. \\
\hline & $\begin{array}{l}\text { Reducir la oferta de } \\
\text { plazas }\end{array}$ & $\begin{array}{l}\text { Ofertar un número de plazas coherente con las necesidades } \\
\text { del mercado, de forma que se garantice la empleabilidad } \\
\text { de los graduados. }\end{array}$ \\
\hline & Más optatividad & $\begin{array}{l}\text { Mayor oferta de optativos que permita al estudiante } \\
\text { reconducir su formación a distintos itinerarios acordes a } \\
\text { sus intereses profesionales. }\end{array}$ \\
\hline & Prácticas externas & $\begin{array}{l}\text { Mayor durabilidad del periodo de prácticas externas, } \\
\text { mayor cantidad y en distintos momentos y lugares } \\
\text { (cambiantes) (subcódigos). }\end{array}$ \\
\hline & $\begin{array}{l}\text { Coordinación y } \\
\text { continuidad entre } \\
\text { asignaturas }\end{array}$ & $\begin{array}{l}\text { Las asignaturas están coordinadas y siguen una secuencia } \\
\text { lógica para generar el aprendizaje significativo en el } \\
\text { graduado. }\end{array}$ \\
\hline & $\begin{array}{l}\text { Actualización entre } \\
\text { asignaturas }\end{array}$ & $\begin{array}{l}\text { El contenido de las asignaturas debe responder a las } \\
\text { demandadas socio-profesionales del momento actual. }\end{array}$ \\
\hline & $\begin{array}{l}\text { Estudio de } \\
\text { demandas } \\
\text { profesionales }\end{array}$ & $\begin{array}{l}\text { La realización de estudios que permitan examinar la } \\
\text { demanda profesional y diseñar planes formativos } \\
\text { adecuados a estos requerimientos. }\end{array}$ \\
\hline & $\begin{array}{l}\text { Contraste de } \\
\text { opiniones }\end{array}$ & $\begin{array}{l}\text { Contemplar y contrastar la opinión de todos los sectores de } \\
\text { la comunidad educativa para el diseño de los planes de } \\
\text { estudio. Especialmente de alumnos, empleadores y } \\
\text { docentes. }\end{array}$ \\
\hline & $\begin{array}{l}\text { Mayor formación } \\
\text { en CT }\end{array}$ & $\begin{array}{l}\text { Aumentar el desarrollo de estas competencias trasversales } \\
\text { en el currículo universitario, bien con la creación de } \\
\text { asignaturas específicas, bien a través de contenidos } \\
\text { específicos dentro de las asignaturas ya existentes. } \\
\text { Concretamente, se deben reforzar las competencias: de } \\
\text { lengua extranjera, comunicación, investigadora, } \\
\text { motivación, uso de las TIC, control emocional, interacción } \\
\text { social y creatividad (subcódigos). }\end{array}$ \\
\hline \multirow{3}{*}{$\begin{array}{l}\text { Categoría 1.2. } \\
\text { Orientación } \\
\text { profesional }\end{array}$} & $\begin{array}{l}\text { Técnicas de } \\
\text { búsqueda activa de } \\
\text { empleo }\end{array}$ & $\begin{array}{l}\text { Incorporar al currículo, formación sobre técnicas o } \\
\text { herramientas de búsqueda activa de empleo para favorecer } \\
\text { la inserción laboral y empleabilidad del graduado. }\end{array}$ \\
\hline & $\begin{array}{c}\text { Información y } \\
\text { orientación } \\
\text { académica- } \\
\text { profesional }\end{array}$ & $\begin{array}{l}\text { Mayor información y orientación sobre becas, estudios, } \\
\text { salidas profesionales a los estudiantes de grado. }\end{array}$ \\
\hline & Emprendimiento & $\begin{array}{l}\text { Dar a conocer las pautas para construir tu propia empresa } \\
\text { y emprender profesionalmente. }\end{array}$ \\
\hline
\end{tabular}




\begin{tabular}{|c|c|c|}
\hline \multirow{5}{*}{$\begin{array}{l}\text { Categoría 1.3. } \\
\text { Planificación de } \\
\text { la enseñanza }\end{array}$} & $\begin{array}{l}\text { Modalidad de } \\
\text { enseñanza práctica }\end{array}$ & $\begin{array}{l}\text { Clases con carácter práctico basadas en contenidos útiles } \\
\text { para el desempeño profesional del egresado. }\end{array}$ \\
\hline & $\begin{array}{l}\text { Metodologías } \\
\text { activas }\end{array}$ & $\begin{array}{l}\text { Forma de enseñar dinámica centrada en el estudiante y en } \\
\text { su participación en el propio aprendizaje, además de en } \\
\text { alternación con diferentes estrategias que favorezcan el } \\
\text { entendiendo del currículo académico desde distintas } \\
\text { perspectivas. }\end{array}$ \\
\hline & $\begin{array}{l}\text { Experiencias } \\
\text { agentes externos }\end{array}$ & $\begin{array}{l}\text { Traer a clase profesionales del ámbito laboral para que } \\
\text { cuenten sus experiencias y sirvan de ejemplo a los } \\
\text { graduados. }\end{array}$ \\
\hline & Profesorado & $\begin{array}{l}\text { Actualización, compromiso y competencia pedagógica o } \\
\text { habilidades docentes (subcódigos). }\end{array}$ \\
\hline & $\begin{array}{l}\text { Evaluación por } \\
\text { competencias }\end{array}$ & $\begin{array}{l}\text { La evaluación no solo debe valorar conocimientos, sino } \\
\text { también valorar actitudes y valores en los estudiantes, es } \\
\text { decir, competencias. }\end{array}$ \\
\hline
\end{tabular}

Después de elaborar este listado de categorías, se pasa a establecer una relación interpretativa entre ellas para dar un sentido más profundo a las propuestas de mejora establecidas por el alumnado. Para ello, se elabora una red semántica o de contenido, representada en la figura 5 y a continuación se interpreta.

Figura 5. Red de contenido. Propuestas de mejora para ajustar la formación a las demandas profesionales.

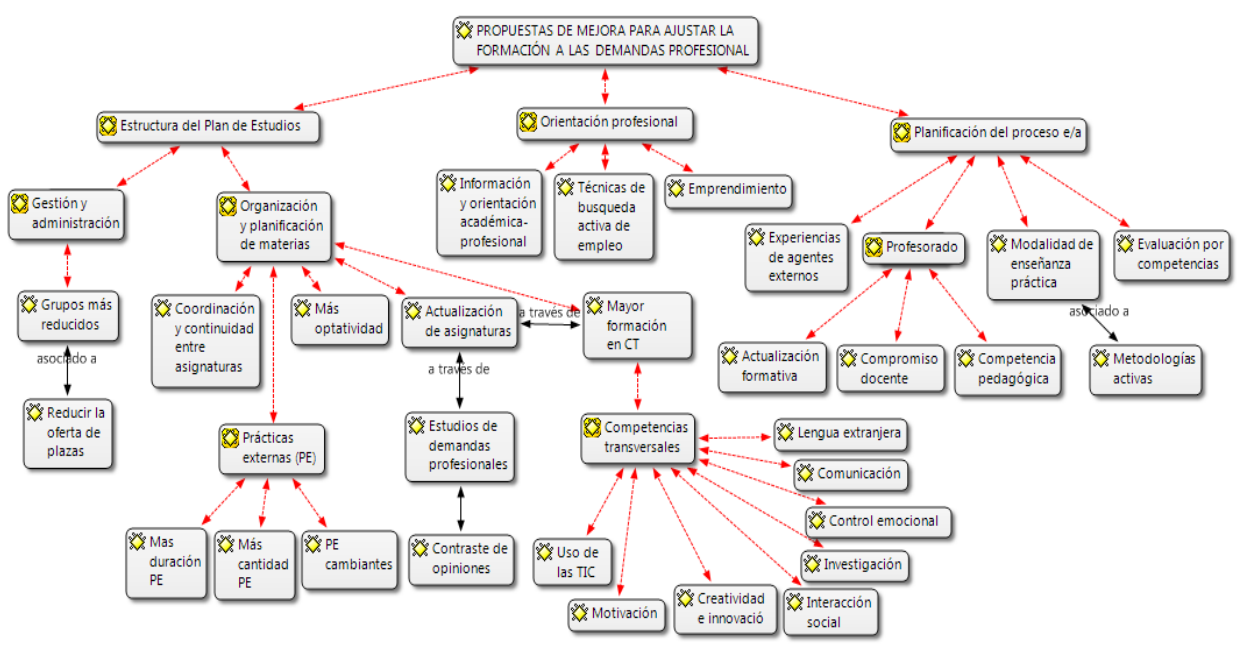


Los códigos que integra la estructura del plan de estudios se dividen a su vez en dos subcategorías. La primera integra aspectos de gestión y administración como crear grupos de alumnos más reducidos o realizar una oferta de plazas más pequeña ${ }^{1}$ acorde con las necesidades del mercado. Los estudiantes, en consonancia con estudios internacionales (OIT, 2015), manifiestan que un elevado número de egresados salen a un mercado laboral con dificultades para absorber la cantidad de profesionales titulados. La segunda subcategoría, denominada organización y planificación de materias, contempla la importancia que el estudiante concede a las prácticas externas, solicitando mayor cantidad, una mayor durabilidad e incluso que estas sean en distintos momentos y rotacionales para ver otros puestos de trabajo, por ejemplo, los estudiantes del grado de Educación Infantil mejorarían su practicum, trasladando sus prácticas escolares al periodo de adaptación de los niños al comienzo del curso escolar.

Por otro lado, las optativas cobran especial protagonismo para acortar distancia entre universidad y mercado, siendo el aumento de estas en el plan de estudios, otras de las mejoras que se plantea pues advierten que con ellas se crea una mayor especialización y están más orientadas al ámbito profesional, de ahí que los estudiantes soliciten ampliar su oferta, acorde con sus intereses profesionales.

La coordinación entre asignaturas para evitar solapamientos y generar continuidad entre las mismas con vistas a la consecución de un aprendizaje significativo, es una de las mejoras más retiradas por los estudiantes. A esta se le puede sumar la actualización permanente de los contenidos para llevar a cabo una formación relevante significativa para afrontar la realidad sociolaboral. Para ello proponen a su vez realizar estudios de demandas profesionales donde se contemple la visión de los empleadores y se analizan las necesidades formativas, junto con los agentes educativos y ellos mismos.

A esta iniciativa, se suma el incremento de la formación en competencias transversales. El estudiante percibe una carencia formativa en este sentido que debe potenciarse a través de asignaturas concretas (como es el caso de incorporar a todos los currículos una asignatura obligatoria de conocimiento de una lengua extranjera) o bien con contenidos específicos a lo largo de la carrera, enfocados principalmente al desarrollo de la competencia comunicativa e interacción social, investigadora, motivacional, de control emocional, uso de las TIC y creatividad.

La segunda categoría integra propuestas de mejoras dirigidas a la planificación del proceso de enseñanza y aprendizaje. Es el caso de la incorporación e invitación de agentes externos como expertos o profesionales en ejercicio a las aulas. Además, los estudiantes solicitan al profesorado una mayor actualización de sus competencias, especialmente sus habilidades docentes, y un mayor compromiso e implicación con el proceso educativo.

Junto a las propuestas dirigidas a reforzar la competencia profesional del docente, el ajuste formativo se relaciona con una modalidad de enseñanza práctica basada en contenidos útiles y aplicables (conocimientos teóricos que vayan acompañados de más prácticas o más aplicaciones prácticas de la teoría) y el uso de una metodología de enseñanza basada en estrategias activas, donde se potencie la participación del estudiante en el aula, y en su propio aprendizaje.

Por último, en esta segunda categoría se integra la importancia de evaluar no solo conocimientos, sino también actitudes y valores, tal y como expresan, no es solo importante

El texto en cursiva corresponde a citas textuales de los participantes. 
conocer si el estudiante tiene el conocimiento teórico. La actitud de una persona y sus valores son aspectos clave para su desarrollo y éxito profesional.

La tercera categoría subraya la importancia de potenciar la orientación profesional en las instituciones universitarias. Concretamente las propuestas de mejora realizadas se dirigen hacia una mayor información sobre becas, estudios posteriores y especialmente salidas profesionales del título que están cursando, así como formación en técnicas de búsqueda de empleo como por ejemplo funcionamiento de las bolsas de trabajo o el emprendimiento.

Entre estas mejoras se encuentra el incremento de una formación basada en competencias transversales con la que se pretende conseguir una mayor percepción de ajuste formativo de la universidad a los requerimientos profesionales/laborales.

\section{DISCUSIÓN Y CONCLUSIONES}

El cambio estructural producido de los sistemas universitarios con la implantación del Espacio Europeo de Educación Superior surge de la necesaria evolución de la formación en paralelo a los avances tecnológicos en el ámbito productivo. Sin embargo, todavía hoy se aprecian dificultades en el rendimiento de las universidades y una brecha significativa entre formación y mercado de trabajo.

Los resultados muestran una percepción de adecuación formativa a las necesidades laborales por debajo de la deseada. Tan solo el $28 \%$ del alumnado la percibe como bastante o muy adecuada. Igualmente, las diferencias entre ramas de conocimiento son destacables, siendo los estudiantes de Ingeniería y Ciencias de la Salud quienes perciben un acercamiento mayor entre sus perfiles formativos y profesionales. Por el contrario, los estudiantes de los grados de Artes y Humanidades perciben esta adecuación más baja. Estos resultados son coherentes con el estudio realizado por Michavila et al. (2016) desde la visión de los graduados ya insertados en el mercado de trabajo a escala nacional, coincidiendo este mayor ajuste formativo en el área de Ciencias de la Salud y el menor, en Artes y Humanidades.

Asimismo, resulta curioso relacionar ese resultado en la adecuación formativa con las tasas de desempleo de los estudiantes universitarios. Las titulaciones técnicas y de Ciencias de la Salud son las que tienen el índice más bajo de desempleo frente a la rama de Artes y Humanidades que presentan el más alto, lo que lleva a pensar en la estrecha relación que existe entre la adecuación de la formación y la inserción laboral, tal y como muestran los estudios de Bartlett y Uvalić (2019), García-Blanco y Cárdenas-Sempértegui (2018), Pérez García (2018) y Teicheler (2015) que señalan que una formación adecuada parece incrementar las posibilidades de empleabilidad de los graduados.

Sin embargo, los estudiantes tienen una visión de adecuación de su formación por debajo de las posibilidades reales que les proporciona desenvolverse en el ámbito profesional. La activación de las competencias adquiridas bien durante la carrera, en el momento de enfrentarse a la realidad laboral o bien, durante las prácticas curriculares externas, muestran un ajuste formativo más elevado. El $42 \%$ del alumnado manifiesta una aplicación alta de su formación a los requerimientos de su ocupación. Un dato más alentador que el de percepción sobre adecuación y más próximo al 77.37\% de los egresados españoles que consideran que su último empleo estuvo bastante o muy relacionado con sus estudios (Michavila et al., 2016). 
Cabe destacar que la aplicación formativa más relevante se encuentra, de forma general, asociada a las enseñanzas técnicas y a las profesiones vinculadas al ámbito comunitario, siendo los estudiantes de Ciencias de la Salud quienes lideran este resultado. Los estudiantes de Ciencias Sociales optan en su mayoría por el ámbito educativo para su desempeño profesional, sin embargo, perciben la aplicación de su formación más elevada en el sector comunitario. Los estudiantes de Artes y Humanidades eligen el ámbito educativo como principal objetivo profesional pero la activación de sus competencias es mayor en el ámbito empresarial. Por último, el alumnado de Ingeniería y los de Ciencias Experimentales se inclinan por el ámbito empresarial mientras consideran la aplicación más elevada en otros ámbitos laborales sin especificar o categorizar por esta investigación y que sería de gran interés profundizar en ellos en próximas investigaciones, de igual manera que de forma más específica en el resto de las titulaciones.

Aunque muchas de las diferencias entre aplicabilidad y ámbito profesional no son significativas, si es preciso tenerlas en cuenta para valorar la tendencia de activación de las competencias desarrolladas en los distintos ámbitos laborales y reflexionar consecuentemente para reconducir y mejorar su formación en función de los itinerarios de inserción a los que optan los graduados.

Las propuestas que plantean los estudiantes para mejorar este desequilibrio o desajuste formativo detectado son muchas y de diversa índole. Todas ellas han sido clasificadas en función de tres categorías principales, que señalan las líneas de acción prioritarias, agrupándose en relación a la estructura del plan de estudios, la planificación del proceso enseñanza y aprendizaje y la orientación profesional que reciben a lo largo de su carrera.

En relación a la primera categoría, las propuestas se dirigen mayoritariamente a la organización y actualización de las asignaturas. Se plantea de forma más concreta mejorar la coordinación para evitar solapamientos o bien, actualizar las competencias dando un mayor protagonismo a las transversales en los currículos universitarios. Estas propuestas están en consonancia con los resultados obtenidos en el proyecto DEHEMS (Pavlin, 2011) que indica la importancia de atender a las nuevas tendencias del mercado, así como a sus oportunidades emergentes desde el desarrollo de las competencias transversales en los currículos universitarios. En esta línea, los futuros graduados de la Universidad de Murcia proponen reforzar su formación más genérica y transversal para garantizar la durabilidad de su formación y capacidad de adaptación a un entorno sociolaboral en permanente cambio.

A esta propuesta cabe añadir la necesidad de cooperación y participación de los distintos grupos sociales en el diseño adecuado y actualizado de los títulos de Grado. Ante esta necesidad, manifiestan la importancia de actualizar el contenido de las asignaturas desde el diálogo continuo con los empleadores, siendo múltiples y diversas las investigaciones que muestran los beneficios del intercambio de percepciones y el diálogo social; la OCDE (2011), Tuyet (2014), Bartlett y Uvalić, (2019), González y Wagenaar (2003), Alonso, Fernández y Nyssen (2009), Martín, Rabadán y Hernández (2013), y Humberg et al. (2013); reconocen como una de las causas del Skill mismatch, la falta de comunicación entre agentes sociales y considera la necesidad de remar en la misma dirección para obtener un mayor conocimiento del mercado laboral y de la formación universitaria respectivamente. En concreto, Palmer, Montaño y Palou (2009) plantean la necesidad de la complementariedad de perspectivas, entre docentes, graduados y empleadores y/o colegios profesionales en la identificación de las competencias a desarrollar en la formación universitaria. Las competencias "no pueden 
ser determinadas unidireccionalmente por la universidad sin tener en cuenta las entidades destinatarias como son las organizaciones laborales" (Alonso-Martín, 2010, p. 86).

Según datos de la Comisión Europea (2010) a través del estudio de Eurobarometer, el $50 \%$ de los empleadores no han contribuido nunca a la discusión sobre el diseño curricular de las titulaciones, tan solo el 6\% afirma haber participado. Sin embargo, el $48 \%$ considera muy o bastante importante su participación. Este porcentaje es más elevado entre los empleadores españoles, donde asciende al 59\% de los encuestados.

Por otra parte, el alumnado considera que una mayor oferta de optativas les puede ayudar de definir su perfil formativo y especialización, así como un aumento de las prácticas externas durante los estudios. En relación a este último aspecto Martínez Clares y Echeverría (2009); Perrenoud (2004) y Tejada (2012) subrayan la importancia de la práctica en la formación por la superioridad que confiere para la adquisición de competencias al ser indisociables de su puesta en marcha. Las prácticas en el currículo enfocan el proceso educativo hacia la dirección correcta, basada en la acción participante y la interacción con el medio (Le Boterf, 1995). En la investigación presente, se constata cómo en aquellas ramas de conocimiento con un perfil formativo tradicionalmente más práctico como son las Ciencias de la Salud y las ingenierías presentan una mayor percepción de ajuste entre la formación y empleo.

Los estudiantes centran gran parte de sus propuestas de mejora en la planificación del proceso de enseñanza y aprendizaje, concretamente proponen aplicar metodologías más activas que generen en el estudiante una mayor implicación en su aprendizaje. Gargallo, Morera y García (2015); Martínez Clares y González Morga (2018a) constatan cómo estas metodologías son más apropiadas para el desarrollo de una formación basada en competencias, puesto que la formación precisa de momentos de acción, pero también de reflexión curricular para evitar que se convierta en una pieza suelta del sistema formativo y deje de cumplir la función que tiene encomendada (Zabalza, 2013).

Igualmente, plantean aumentar el uso de instrumentos de evaluación que visibilicen las competencias adquiridas por el alumnado y no tanto sus conocimientos técnicos o puramente cognitivos. Como afirma Cobo y Moravec (2011) muchos de los instrumentos tradicionalmente utilizados, como las pruebas tipo test, evalúan conocimientos o contenidos dictados por el profesor o que son contemplados en el libro de texto, lo que a su vez estimula, la repetición, memorización o el también llamado aprendizaje bulímico (Llorens, 2013) y penaliza el error junto con la posibilidad de equivocarse como pieza clave en el desarrollo de la creatividad y creación de nuevos aprendizajes.

Por último, exponen potenciar la formación y compromiso docente en la línea que advierte Hagreaves y Fullan (2014) a través de una actitud abierta hacia los cambios producidos tanto dentro como fuera de las instituciones. Para que una formación tenga la efectividad suficiente se necesitan docentes formados en recursos y metodologías para afrontar el nuevo paradigma educativo basado en competencias. Son los mismos docentes quienes reiteran sus dificultades para gestionar el enfoque por competencias, aludiendo a la falta de formación sobre las metodologías más adecuadas y herramientas de evaluación (Martín, 2009 o Villardón-Gallego, 2015).

Al margen de la estructura del plan de estudios y el proceso de enseñanza-aprendizaje, los estudiantes conforman con sus respuestas un tercer bloque de propuestas que alude a la ampliación de los servicios de orientación profesional con especial atención en ampliar la formación e información sobre salidas profesionales y búsqueda activa de empleo. A 
través de la orientación profesional se proporcionan información sobre salidas y perfiles profesionales y el estudiante se vuelve consciente de sus carencias formativas que podrán reconducirse y solventarse desde la intervención orientadora, como subraya el estudio de Eurydice (2014).

Toda investigación presenta limitaciones, reflexionar sobre ellas ayuda a realizar un ejercicio de prospección y mejora de dicha investigación. En este caso, el presente trabajo contempla datos de una única Universidad por lo que se debe ser prudente a la hora de generalizar sus resultados a otras universidades. A pesar de un marco teórico relevante para el tema objeto de estudio y el desarrollo de una metodología de investigación rigurosa, las diferencias contextuales y personales que caracterizan a las diferentes instituciones pueden influir de modo distinto en los resultados. No obstante, el hecho de centrarse en una única realidad universitaria para analizar el ajuste formativo se considera para otros autores un valor añadido, Gaebel, Hauschildt, Mühleck y Smidt (2012) señalan que el motivo por el que las implicaciones de los resultados obtenidos en otros estudios de similares características no hayan sido suficientes es precisamente por el hecho de no centrarse en una única institución. Cuanto más acotado está la realidad estudiada, mayor implicación genera en su comunidad, más fácil resulta aplicar las propuestas de mejora y en definitiva que estas reviertan en el diseño, planificación y rendimiento del sistema.

\section{REFERENCIAS BIBLIOGRÁFICAS}

Alonso, L., Fernández, C. J. y Nyssen, J. M. (2009). El debate sobre las competencias: Una investigación cualitativa en torno a la educación superior y el mercado de trabajo en España. Madrid: ANECA.

Alonso-Martín, P. (2010). La importancia y el nivel de desarrollo de las competencias en Psicología. Psicología desde el Caribe, (25), 84-107.

Bartual, M. T. y Turmo, J. (2016). Educación superior y competencias para el empleo. El punto de vista de los empresarios. Revista Complutense de Educación, 27(3), 1211-1228. doi: 10.5209/ rev_RCED.2016.v27.n3.47645

Bartlett W. y Uvalić M. (2019). Educación superior y mercado laboral graduado en los Balcanes Occidentales. En: Osbild R., Bartlett W. (eds) Economías de los Balcanes Occidentales en transición. Sociedades y órdenes políticos en transición. Springer, Cham. doi: 10.1007/978-3319-93665-9_4

Bauman, Z. (2007). Los retos de la educación en la modernidad líquida. Barcelona, España: Gedisa. Centro Europeo para el Desarrollo de la Formación Profesional [CEDEFOP] (2014). Desajuste de competencias: Más de lo que parece a simple vista. Nota informativa. Recuperado de https:// www.sepe.es/contenidos/personas/formacion/refernet/pdf/NI-032014_es.pdf

[CEDEFOP] (2015). Skills, qualifications and jobs in the EU: The making of a perfect match? Luxemburgo: Oficina de Publicaciones de la Unión Europea.

[CEDEFOP] (2018). 2018 European Skills Index. Recuperado de https://skillspanorama. cedefop.europa.eu/en/useful_resources/2018-european-skills-index-technical-report

. [CEDEFOP] (2020). Europa ante el reto de las competencias. Recuperado de https://www. cedefop.europa.eu/files/9068_es.pdf

Cobo, C. y Moravec, J. (2011). Aprendizaje invisible. Hacia una nueva ecología de la educación. Barcelona, España: UBe.

Comisión Europea (2010). Employers' perception of graduate employability. Luxemburgo: Oficina de Publicaciones de la Unión Europea. 
Eurydice (2014). Modernización de la Educación Superior en Europa: Acceso, permanencia y empleabilidad 2014. Informe Eurydice. Luxemburgo: Oficina de Publicaciones de la Unión Europea.

Franke, M. (2016). La educación y la formación profesional. Fichas técnicas sobre la Unión Europea. Parlamento Europeo. Recuperado de http://bit.ly/2knGz5Y

Freire, M. J., Teijeiro, M. M. y Pais, C. (2013). La adecuación entre las competencias adquiridas por los graduados y las requeridas por los empresarios. Revista de Educación, 362, 13-41. doi: 10.4438/1988-592X-RE-2011-362-151

Gaebel, M., Hauschildt, K., Mühleck, K. y Smidt, H. (2012). Tracking learners' and graduates' progression paths (Trackit). Bruselas, Bélgica: European University Association.

García-Blanco, M. y Cárdenas-Sempértegui, E. B. (2018). La inserción laboral en la Educación Superior. La perspectiva latinoamericana. Educación XX1, 21(2), 323-347, doi: 10.5944/ educXX1.16209

García-Aracil, A. y Van der Velden, R. (2008). Competencies for young European higher education graduates: Labor market mismatches and their payoffs. Higher Education, 55(2), 219-239. doi: 10.1007/s10734-006-9050-4

Gargallo, B., Morera, I. y García, E. (2015). Metodología innovadora en la universidad. Sus efectos sobre los procesos de aprendizaje de los estudiantes universitarios. Anales de Psicología, 31(3), 901-015. doi: 10.6018/analesps.32.1.179871

González, J. y Wagenaar, R. (2003). Tuning educational structures in Europe. Final report. Phaseone. Bilbao, España: Universidad de Deusto.

Hargreaves, A. y Fullan, M. (2014). Capital profesional. Madrid, España: Morata.

Humberg, M., Van der Valden, R. y Verhagen, A. (2013). The employability of higher education graduates: The employers' perspective. Maastricht, Países Bajos: Oficina de Publicaciones de la Unión Europea

Le Boterf, G. (1995). De la competénce: essai sur un attracteur étrange. París, Francia: Les Editions d'Organisations.

Llorens, F. (Coords.) (2013). Tendencias Universidad. En pos de la educación activa. Madrid: Cátedra UNESCO de Gestión y Política Universitaria, Universidad Politécnica de Madrid.

Martín, E. (2009). Profesorado competente para formar alumnado competente: El reto del cambio docente. En J. I. Pozo y M. P. Echeverría (Coords.), Psicología del aprendizaje universitario: La formación en competencias (pp. 199-215). Madrid, España: Morata.

Martín, M., Rabadán, A. B. y Hernández, J. (2013) Desajustes entre formación y empleo en el ámbito de las enseñanzas técnicas universitarias: La visión de los empleadores de la Comunidad de Madrid. Revista de Educación, (360), 244-267. doi: 10-4438/1988-592X-RE-2011-360-110

Martínez Clares, P. y Echeverría, B. (2009). Formación basada en competencias. Revista de Investigación Educativa, 27(1), 125-147.

Martínez Clares, P. y González Morga, N. (2018a). Metodologías de enseñanza en la universidad y su relación con el desarrollo de competencias transversales. Cultura y Educación, 30(2), 233-275.

. (2018b). Las competencias transversales en la universidad: propiedades psicométricas de un cuestionario. Educación XX1, 21(1), 231-262, doi: 10.5944/ educXX1.20194

Michavila, F., Martínez, J. M., Martín-González, M., García-Peñalvo, F. J. y Cruz-Benito, J. (2016). Barómetro de empleabilidad y empleo de los universitarios en España, 2015 (Primer informe de resultados). Madrid, España: Observatorio de Empleabilidad y Empleo Universitarios.

Organización Internacional del Trabajo [OIT] (2014). Skills mismatch in Europe. Ginebra, Suiza: Autor. Organización para la Cooperación y el Desarrollo Económicos [OCDE] (2011). Towards an OECD Skills Strategy. Recuperado de http://www.oecd.org/edu/47769000.pdf

[OCDE] (2012). Better Skills, Better Jobs, Better Lives, OECD Publishing. Recuperado de http://www.skillsforemployment.org/wcmstest4/groups/skills/documents/skpcontent/ddrf/ mduw/ edisp/wcmstest4_050446.pdf 
[OCDE] (2015). OECD Skills Strategy Informe de Diagnóstico. España: Autor. Recuperado de http://www.oecd.org/skills/nationalskillsstrategies/Spain_Diagnostic_Report_Espagnol.pdf

Organización Internacional del Trabajo [OIT] (2015). Tendencias mundiales del empleo juvenil 2015. Promover la inversión en empleos decentes para los jóvenes. Ginebra, Suiza: Autor.

Osses, S., Sánchez, I. e Ibáñez, F. M. (2006). Investigación cualitativa en educación: Hacia la generación de teoría a través del proceso analítico. Estudios pedagógicos, 32(1), 119-133.

Palmer, A., Montaño, J. y Palou, M. (2009). Las competencias genéricas en la educación superior: Estudio comparativo entre la opinión de empleadores y académicos. Psicothema, 21(3), 433438.

Pavlin, S. (2011). DEHEMS. Network for the development of higher education management systems. Varieties of professional domains and employability determinants in higher education. Bolonia, Italia: AlmaLaurea.

Pérez García, F. (Dir.) (2018). Itinerarios de inserción laboral y factores determinantes de la empleabilidad. Formación universitaria versus entorno. Bilbao: Fundación BBVA.

Perrenoud, P. (2004). Diez nuevas competencias para enseñar. Madrid, España: Graó.

Rodriguez, C., Lorenzo, O. y Herrera, L. (2005). Teoría y práctica del análisis de datos cualitativo. Proceso general y criterios de calidad. Revista Internacional de Ciencias Sociales y Humanidades, 15(2), 133-154.

Rodríguez-Esteban, A., Vidal, J. y Vieira, M.-J. (2019). Un análisis de la empleabilidad de los universitarios en España a través del ajuste horizontal. Revista de Educación, (384), 229-254. doi:10.4438/1988-592X-RE-2019-384-411

Schwab, K. (2016). The fourth industrial revolution. Ginebra, Suiza: World Economic Forum.

Strauss, A. y Corbin, J. (2002). Bases de la investigación cualitativa: técnicas y procedimientos para desarrollar la teoría fundamentada. Medellín, Colombia: Universidad de Antioquia.

Teichler, U. (2015). Changing perspectives: The professional relevance of higher education on the way towards the highly-educated society. European Journal of Education, 50(4),1465-3435. doi:10.1111/ejed.12146

Tejada, J. (2012). La alternancia de contextos para la adquisición de competencias profesionales en escenarios complementarios de educación superior: Marco y estrategia. Educación XX1, 15(2), 17-40. doi: 10.5944/educxx1.15.2.125

Tuyet, T. (2014). Is graduate employability the 'whole-of-higher-education-issue'? Journal of Education and Work, 28(3), 207-227. doi:10.1080/13639080.2014.900167

United Nations Educational, Scientific and Cultural Organization [UNESCO]

Ursin, J. (2017). Transforming finnish higher education institutional mergers and conflicting academic identities. Revista de Investigación Educativa, 35(2), 307-316. doi. http://dx.doi.org/10.6018/ rie.35.2.295831

Villardón-Gallego, L. (Coord.) (2015). Competencias genéricas en educación superior. Madrid, España: Narcea.

Zabalza, M. A. (2013). El practicum y las prácticas en empresas en la formación universitaria. Madrid, España: Narcea. 
